\title{
ITS in Ill-Defined Domains: Toward Hybrid Approaches
}

\author{
Philippe Fournier-Viger ${ }^{1}$, Roger Nkambou ${ }^{1}$, Engelbert Mephu Nguifo $^{2}$ and André \\ Mayers $^{3}$ \\ Dept. of Computer Sciences, University of Quebec in Montreal. Camada \\ ${ }^{2}$ Dept. of Mathematics and Computer Sciences, Université Blaise-Pascal Clermont 2, France \\ ${ }^{3}$ Dept. of Computer Sciences, Université of Sherbrooke, Canada \\ fournier_viger.philippe@courrier.uqam.ca,nkambou.roger@uqam.ca, mephu@isima.fr, \\ andre.mayers@usherbrooke.ca
}

\begin{abstract}
Classical approaches for supporting tutoring services face several limitations for ill-defined domains. To overcome these limitations, we argue for the utilization of hybrid approaches for supporting tutoring services. In this paper, we describe a hybrid model that combines an expert system, the modeltracing paradigm, and a data mining approach in an ITS for learning to operate a robotic arm. The result is tutoring services that exceed what was possible to offer with each individual approach for this domain.
\end{abstract}

\section{Introduction}

Domains where classical approaches for building intelligent tutoring systems (ITS) are not applicable or do not work well have been termed "ill-defined domains" [1]. For these domains, classical approaches for supporting tutoring services face several limitations. An example is model-tracing [2], which consists in comparing a predefined task model with learners' solutions. Designing a task model by hand can be very hard and time-consuming for ill-defined domains. A second approach is constraint-based modelling [3]. Although, it is effective for some ill-defined domains, it cannot support tutoring services such as suggesting next problem-solving steps to learners, writing constraints can be difficult and time consuming, and a huge number of constraints can be required [1]. A third approach is to integrate an expert system in an ITS to generate expert solutions or for comparing learner solutions with ideal solutions [1]. Expert systems are appropriate for many ill-defined domains. But not all of them can explain their reasoning to learners. Therefore, we here argue for the use of hybrid approaches for ill-defined domains. The idea is to combine advantages of different approaches to avoid their limitations.

\section{An Hybrid Model in CanadarmTutor}

We illustrate this approach with CanadarmTutor [4], an ITS for learning to operate the Canadarm2 robotic arm, a 7 degrees of freedom, robotic arm, deployed on the International Space Station (ISS). The main learning activity in CanadarmTutor is to 
move the arm from a given configuration to a goal configuration. It is a difficult task since operators do not have a direct view of the scene of operation on the space station and must rely on cameras mounted on the manipulator and at strategic places in the environment where it operates. To move the arm, an operator must select at every moment the best cameras for viewing the scene of operation, select and perform joint rotations for moving the arm, and avoid dangerous situations. The task of moving the arm is ill-defined because even if there are some general rules for moving the arm, there are no clear strategies for choosing joints rotations [6].

To support tutoring services in CanadarmTutor, we have initially applied the "expert system approach" by integrating a special path-planner which is based on a probabilistic roadmap approach [4]. The path-planner can automatically generate correct arm's moves avoiding obstacles, consistent with the best available camera views to achieve a given goal [4]. But, the generated paths are not always realistic or easy to follow, as they are not based on human experience, and they do not cover aspects of the task such as how to select/adjust cameras. Also, it cannot support tutoring services such as estimating knowledge of learners as there is no knowledge or skills representation.

To overcome these limitations, we applied the "model-tracing approach" [5]. To do so, we used a custom cognitive model, which is designed for taking into account aspects of spatial reasoning. With this cognitive model, we modelled the main steps for moving the arm as a set of rules with declarative knowledge. This modeling permits CanadarmTutor to automatically evaluate a learner's spatial representations and skills during arm manipulation, and generate personalized feed-back (see [5] for details). Although the task model specified by hand provided a fine cognitive assessment of a learner's knowledge for the main steps of the manipulation task, it does not go into finer details such as how to select joint rotations for moving Canadarm2. The reason is that at this level of details, it is very difficult to define a task model for generating the joint rotations that a human would execute.

To avoid this limitation, we developed a novel approach for supporting tutoring services for ITS [6]. It consists of recording user solutions for a task and then to apply a custom data mining algorithm for automatically extracting part of solutions that occur frequently. The idea is that even if there is a huge number of possibilities for a task and no clear strategies for finding solutions, there may be some parts of solutions that appear frequently and can be used for supporting tutoring services such as suggesting next problem-solving steps. We have applied this approach in CanadarmTutor and obtained what we call "partial task models". This successfully allows CanadarmTutor to offer tutoring services such as suggesting joint rotations for moving the arm. Recently we have extended this approach by annotating user solutions with contextual information (skills required to perform the solution, success, expertise level, etc.), so that partial task models contain this information [6]. This is a very useful feature, as it allows for example to discover frequent parts of solutions that are common to experts possessing a particular skill, and that have completed the exercise successfully. This is used in CanadarmTutor to evaluate learner profiles based on the solution paths that they follow [6]. Although learning partial task models from user solutions in CanadarmTutor allows providing useful help to learners at the level of joint manipulations - which was impossible to achieve with the cognitive 
model or the path-planner [6], one problem is that no help can be offered to learners if part of a solution path was previously unexplored by other users.

We therefore took the decision to combine the three approaches in a hybrid model in CanadarmTutor. Because of space limitation, we only describe its main features, here. First, when a learner clicks on "what I should do next?" during an exercise, CanadarmTutor offers advices on the general procedure for moving the arm thanks to the cognitive model and suggest joint rotations based on the partial task models. If no help can be offered with the partial task model for the current task, a path is generated with the path planner and presented to the learner.

Second, the skills from the cognitive model are now used to annotate the recorded solutions taken as input by the data mining algorithm. Therefore partial task models now include skills from the cognitive model. CanadarnTutor uses this new information to assess skills of the cognitive model by looking at the solution path followed by the learner according to the partial task model. For example, if a learner follows several patterns demonstrated by people with a particular skill, CanadarmTutor will raise its confidence that the learner possesses the skill.

Other tutoring services offered thanks to the hybrid model are generating exercises tailored to the learner (cognitive model), generating demonstration (cognitive model + path planner + partial task models), offering proactive help about camera selection (cognitive model) and letting the learner explore freely the domain knowledge (cognitive model + partial task model). We have performed a preliminary experimentation with the hybrid model and users have been very satisfied.

In conclusion, the tutoring services now offered in CanadarmTutor with the hybrid approach greatly exceed what was possible to offer with each individual approach for the ill-defined task of the robotic arm manipulation. Note that we did not just put together tutoring services provided by the three approaches. Rather, we have integrated each one with each other to provide coherent and rich tutoring services.

\section{References}

[1] Fournier-Viger, P., Nkambou, R., Mephu Nguifo, E.: Supporting tutoring services in ill-defined domains. In: Nkambou et al. (eds.) Advances in intelligent tutoring systems, Springer (2010)

[2] Koedinger, K. R., Anderson, J. R., Hadley, W. H., Mark, M. A.: Intelligent Tutoring goes to school in the big city. Intenational Journal of Artificial Intelligence in Education, 8, 30-43 (1997)

[3] Mitrovic, A., Mayo, M., Suraweera, P., Martin, B.: Contraint-based tutors: a success story. In: Proc. of IEA AIE 2001, pp. 931-940 (2001)

[4] Kabanza, F., Nkambou, R., Belghith, K.: Path-Planning for Autonomous Training on Robot Manipulators in Space. In: Proc. 19th Intern. Joint Conf. Artificial Intelligence (2005)

[5] Fournier-Viger, P., Nkambou, R., Mayers, A.: Evaluating Spatial Representations and Skills in a Simulator-Based Tutoring System. IEEE Transactions on Learning Technologies, 1(1), 63-74 (2008)

[6] Fournier-Viger, P., Nkambou, R., Mephu Nguifo, E.: Exploiting Partial Problem Spaces Learned from Users' Interactions to Provide Key Tutoring Services in Procedural and Ill-Defined Domains. In: Proc. AIED 2009. IOS Press. pp. 383-390 (2009)

Acknowledgments. We thank the Canadian Space Agency, NSERC and FQRNT for their logistic and financial support, and current/ past members of GDACIPLANIART. 